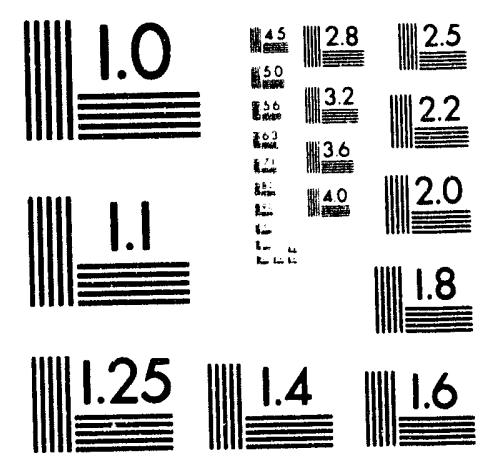



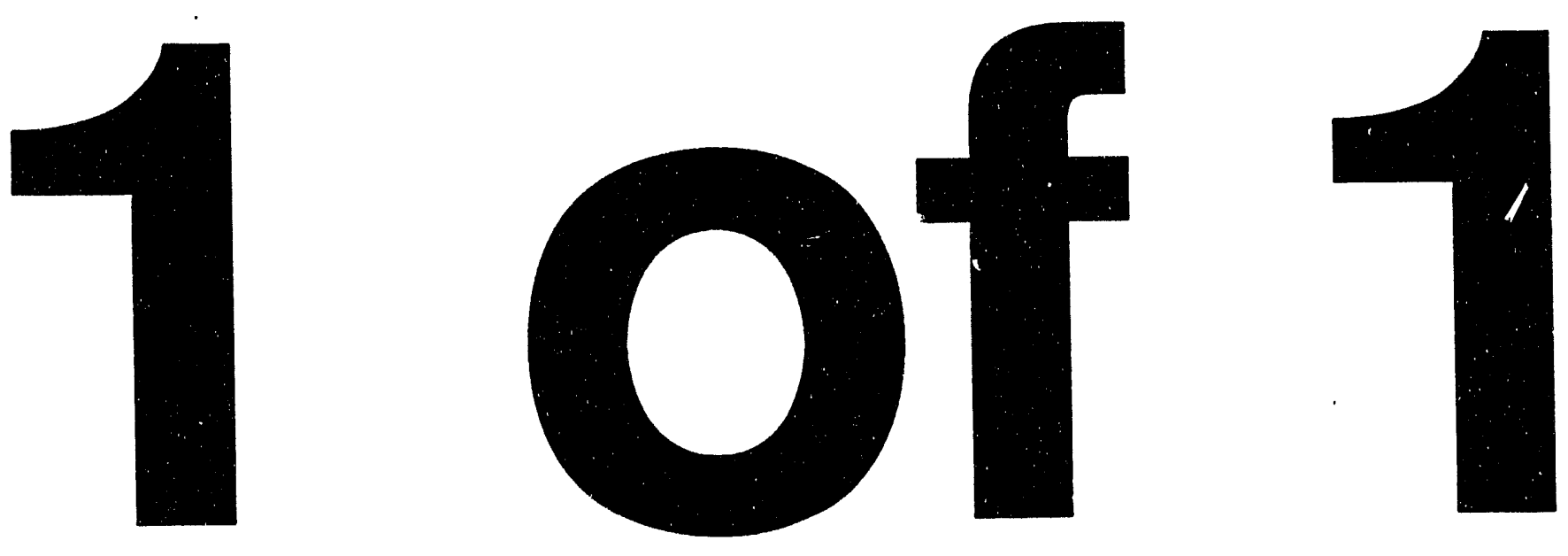
$\operatorname{Cos} 4-930676--47$

LA-UR- $93-3185$
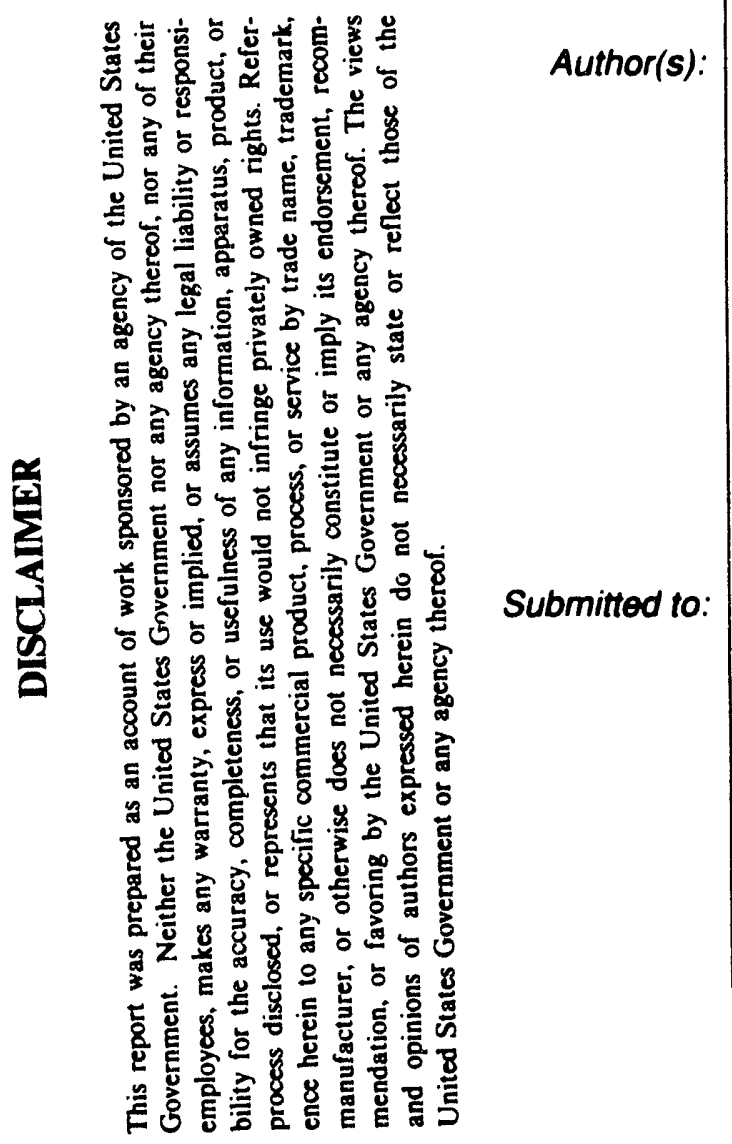

M. S. Shaw, T-14

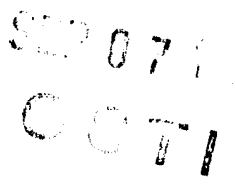

CHEMICAL EQUILIBRIUM IN HIGH PRESSURE MOLECULAR FLUID MIXTURES

Proceedings of the 1993 Joint AIRAPT/APS Topical

Conference on High Pressure Science and Technology

held in Colorado SPrings on June 28-July 2, 1993

\section{MASTER}

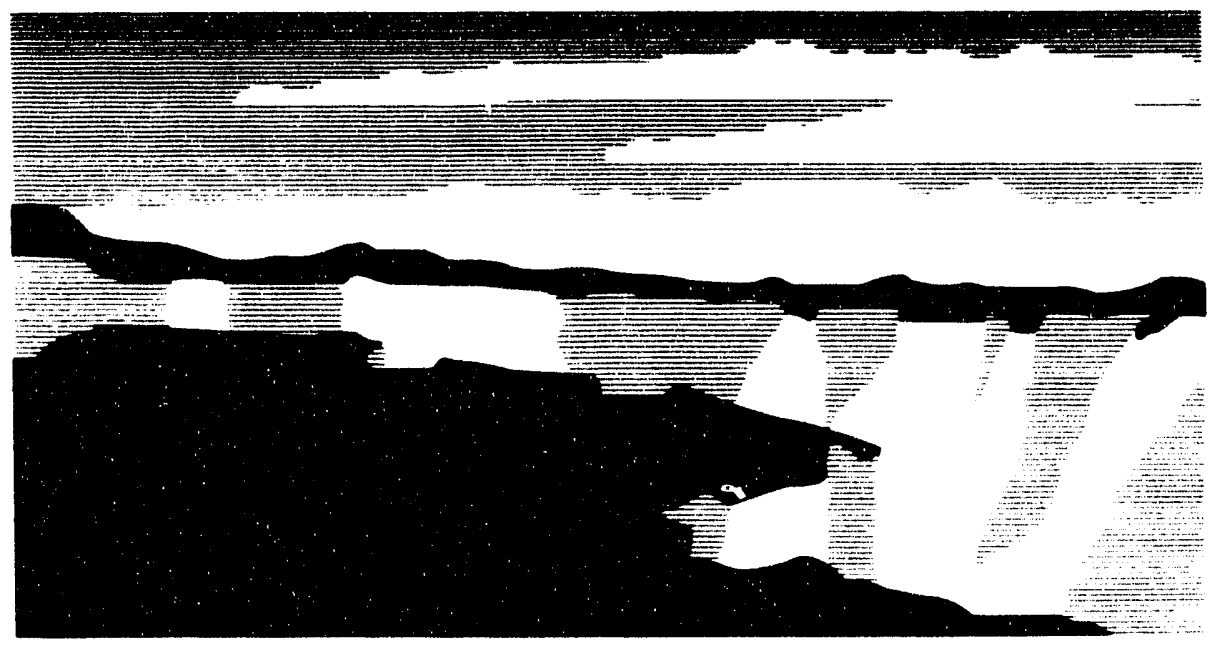

LOS Alamos National Laboratory, an affirmative action/equal oppontunity empldyer, is operated by the University of Calitornia for the U.S. Deparment of Energy under contract W.7405-ENG-36. By acceptance of this article, the publisher recognizes that the U.S. Government retains a nonexclusive, royalty-free license to publish or reproduce the publiated form of this contribution, or to allow others to do so. for U.S. Government purposes. The Los Alamos Najional Laboratory requests that the publisher identity this artich as work pertormed under the auspices of the U.S. Department of Energy. 012 


\title{
CHEMICAL EQUILIBRIUM IN HIGH PRESSLRE MOLECULAR FLUID MIXTURES
}

\author{
M. S. Shaw
}

Group T-14, Mail Stop B214

Los Alamos National Laboratory

Los Alamos, New Mexico 87545

The $\mathrm{N}_{\text {atom. }} \mathrm{PT}$ Monte Carlo simulation method has been reformulated to incorporate multiple species and chemical reactions with changes in the total number of molecules. While maintaining a constant number of each type of atom, the number of molecules is changed by turning on and off the interactions of any particular molecular position with other molecules. Chemical reactions are allowed as a correlated move of atoms to different molecular locations. Equilibrium chemical composition is determined as an average over the simulation along with equation of state quantities. A large set of simulations has been made with the system $\mathrm{N}_{2}+\mathrm{O}_{2} \rightleftharpoons \mathrm{NO}$ covering a wide range in $\mathrm{P}$ and $T$. Both Hugoniot states and the $C J$ point have been determined and are shown to be sensitive to the potentials between unlike species.

\section{INTRODUCTION}

Under shock conditions of sufficient magnitude, most molecular substances will break up into a mixture of smaller molecular and atomic product species. For example, molecules composed of $\mathrm{C}, \mathrm{H}, \mathrm{N}$, and $\mathrm{O}$ (such as many explosives, polymers, etc.), form a 'soup' of products such as $\mathrm{CO}_{2}, \mathrm{H}_{2} \mathrm{O}, \mathrm{N}_{3}, \mathrm{C}, \mathrm{NO}, \mathrm{CO}, \mathrm{O}_{2}$, $C \vec{B}_{4}, N H_{3}, H_{2}$, etc. Given a set of interaction poteratials, there are a variety of well established statisticel mechanics simulation methods ${ }^{1}$ for molecular fluid mixtures of a given composition. Chemical equilibrium composition by standard methods increases the computational cost substantially. With the inclusion of many species, the equilibration process requires a search over a multi-dimensional composition space. At this point, the standard methods become impractical. The author has recently developed the $\mathrm{N}_{\text {atom. }}$ PT Monte Carlo simulation method ${ }^{2,3}$ that includes chemical equilibration as a natural part of the simulation. This paper includes a general reformulation of the method to incorporate multiple species and chemical reactions with changes in the total number of molecules. A large set of simulations has been made with the prototype system $\mathrm{N}_{2}+\mathrm{O}_{2} \rightleftharpoons \mathrm{NO}$ covering a wide range in $\mathrm{P}$ and $T$. Both Hugoniot states and the $C J$ point have been determined and are shown to be sensitive to the potentials between unlike species.

\section{METHOD}

The classical partition function for the atomiccanonical ensemble, with all atoms identical, is just

$$
Q(N, V, T)=\frac{\Lambda^{-3 N}}{N !} Z(N, V, T)
$$

where $N$ is the number of atoms, $\Lambda$ is the de Broglie thermal wavelength and $\mathrm{Z}(\mathrm{N}, \mathrm{V}, \mathrm{T})$ is the configuration integral given by

$$
\begin{aligned}
& Z(N, V, T)= \\
& \int \exp \left[-\beta U\left(\mathbf{r}_{1}, \ldots, \mathbf{r}_{N}\right)\right] d \mathbf{r}_{1} \cdots d \mathbf{r}_{N}
\end{aligned}
$$

With A different types of atoms, $N_{i}$ atoms of type i, and a total of $N=\sum N_{i}$ atoms of all types, the partition function becomes

$$
\begin{aligned}
& Q\left(N_{1}, N_{2}, \cdots, N_{A}, V, T\right)= \\
& \frac{\Lambda_{1}^{-3 N_{1}} \cdots \Lambda_{A}^{-3 N_{A}}}{N_{1} ! \cdots N_{A} !} Z\left(N_{1}, \cdots, N_{A}, V, T\right) .
\end{aligned}
$$

Likewise, the partition function for a molecular mixture, within the approximation of separable molecular internal degrees of freedom, can be written as

$$
\begin{aligned}
& Q\left(M_{1}, \cdots, M_{B}, V, T\right)= \\
& \frac{Z\left(M_{1}, \cdots, M_{B}, V, T\right)}{M_{1} ! \cdots M_{B} ! \Lambda_{1}^{3 M_{1}} \cdots \Lambda_{B}^{3 M_{B}}} q_{1}^{M_{1}} \cdots q_{B}^{M_{B}} .
\end{aligned}
$$

The $q_{i}^{\prime} s$ are those parts of the partition function, such a vibration and rotation, which correspond to an isolated molecule. Equation (4) is the restriction (e.g. the rigid rotor-harmonic oscillator approximation) of Equation (3) to those atomic coordinates which correspond to the chosen molecular mixture composition. Now we can I lax this restriction slightly to include any set of atc nic coordinates which corresponds to any set of $M_{i}^{\prime}=$ which preserves the atomic compositions. Those e.comic coordinates associated with each set of $M_{i}^{\prime} s$ can 
be separately approximated by Equation (4) and the integral over all coordinates allowed by the relaxed restriction becomes a sum of the form

$$
\begin{aligned}
& Q\left(N_{1}, \cdots, N_{A}, V, T\right)= \\
& \sum^{\prime} Q\left(M_{1}, \cdots, M_{B}, V, T\right),
\end{aligned}
$$

where $\Sigma^{\prime}$ indicates only those sets of $M_{i}^{\prime} s$ which preserve the set of $N_{i}^{\prime} s$. Note that each set of $M_{i}^{\prime} s$ samples a different nonoverlapping region of atomic coordinate space due to the different sets of atomic correlations which define the molecules. The atomic-isothermalisobaric ensemble partition function is

$$
\begin{aligned}
& \Delta\left(N_{1}, \cdots, N_{A}, P, T\right)= \\
& \int_{0}^{\infty} \exp (-\beta P V) Q\left(N_{1}, \cdots, N_{A}, V, T\right) d V .
\end{aligned}
$$

A Monte Carlo simulation can be made in these ensembles if a type of move between nonoverlapping regions can be found which is reasonably likely. This can be accomplished by interchanging atoms between molecules. In general, the number of molecules could change. Let $M$ denote the total number of molecules, i.e. $M=\sum_{i=1}^{B} M_{i}$. A change of varibles to a reduced distance $s$ given by

$$
\mathbf{s}_{i}=V^{-\mathbf{s}} \mathbf{r}_{i}
$$

which has the normalization

$$
\int d s_{i}=1
$$

Each molecular $Q$ can be rewritten as

$$
\begin{aligned}
& Q\left(M_{1}, \cdots, M_{B}, V, T\right)= \\
& V^{M} \frac{q_{1}^{M_{1}} \cdots q_{B}^{M_{\bullet}}}{M_{1} ! \cdots M_{B} ! \Lambda_{1}^{3 M_{1}} \cdots \Lambda_{B}^{3 M_{B}}} \\
& \times \int \exp \left[-\beta U\left(s_{1}, \ldots, s_{M}\right)\right] d s_{1} \cdots d s_{M^{\prime}} .
\end{aligned}
$$

Since $\int d s_{i}=1$, integrations over $s_{i}$ were added such that $M^{\prime} \geq M$ for all molecular compositions of iuterest. Then Equation (6) becomes

$$
\begin{aligned}
& \Delta\left(N_{1}, \cdots, N_{A}, P, T\right)= \\
& \int_{0}^{\infty} \int \sum e^{W} d V d s_{1} \cdots d s_{M} \cdots d s_{M^{\prime}},
\end{aligned}
$$

where

$$
\begin{aligned}
& W\left(s_{1}, \ldots, s_{M}, M_{1}, \cdots, M_{B}\right)= \\
& -\beta\left[U\left(s_{1}, \ldots, s_{M}\right)+P V\right]+M \ln V \\
& +\sum_{i=1}^{B}\left[M_{i}\left(\ln q_{i}-3 \ln \Lambda_{i}\right)-\ln \left(M_{i} !\right)\right] .
\end{aligned}
$$

Note that the first two terms on the righthand side are just those of an XPT ensemble with fixed molecular composition.

A Markov chain which gives a limiting distribution proportional to $e^{W}$ is then obtained by accepting a move from state $r$ to states $s$ with the probability $P_{r \rightarrow \text { s given by }}$

$$
P_{r \rightarrow a}=\operatorname{Min}\left[1, \exp \left(W_{t}-W_{r}\right)\right] .
$$

This assumes the usual condition $p_{r \rightarrow g}=p_{s \rightarrow r}$ where the p's indicate the unweighted probability of a move. It is sometimes convenient to relax this condition so that a low probability move is tried less often with a higher chance of being accepted when it is tried. We still want the net flow from $r \rightarrow s$ to be the same as $s \rightarrow r$ at the required distribution. The condition

$$
\exp \left(W_{r}\right) p_{r-a} P_{r \rightarrow s}=\exp \left(W_{s}\right) p_{s \rightarrow r} P_{s \rightarrow r},
$$

leads to the choice

$$
P_{r \rightarrow 0}=\operatorname{Min}\left[1, \exp \left(W_{s}-W_{r}\right) p_{o \rightarrow r} / p_{r \rightarrow g}\right] .
$$

We now have to evaluate the unweighted probabilities of moves for a specific scheme of choosing chemical reactions. The bookkeeping is somewhat easier to follow if we do not keep track of labels for a particular molcule. That is, only the set of positions, $s_{i}$, and corresponding molecule type, $t_{i}$, at each position are used to characterize a state. This indistinguishability of molecules of any given type cancels the $\ln M_{i}$ ! terms from Equation (8) which were originally included to take into account indistinguishability in the usual labeled representation. For $m$ types of molecules allowed in the simulation, let each positive integer through $\mathrm{m}$ denote one molecule type. Also, let $t=0$ be a null particle which keeps track of the extra integration variables introduced in Equation (- $(0)$. A given chemical reaction can be written in the form

$$
\sum_{t=0}^{m} \nu_{t} F_{t} \rightleftharpoons \sum_{t=0}^{m} \xi_{t} F_{t}
$$

where $F_{t}$ is the formula for molecule $t$. For example, the reaction $2 \mathrm{CO}+\mathrm{O}_{2} \rightleftharpoons 2 \mathrm{CO}_{2}$ would be characterized by $F_{0}=$ null, $F_{1}=C O, F_{2}=O_{2}, F_{3}=C O_{2}, \nu_{0}=$ $0, \nu_{1}=2, \nu_{2}=1, \nu_{3}=0, \xi_{0}=1, \xi_{1}=0, \xi_{2}=0, \xi_{3}=2$. Likewise, $\mathrm{N}_{2}+\mathrm{O}_{2} \rightleftharpoons 2 \mathrm{NO}$ would be characterized by $F_{0}=$ null, $F_{1}=N_{2}, F_{2}=O_{2}, F_{3}=N O, \nu_{0}=0, \nu_{1}=$ $1, \nu_{2}=1, \nu_{3}+0, \xi_{0}=0, \xi_{1}=0, \xi_{2}=0, \xi_{3}=2$.

For a given simulation, one or more chemical reactions can be included at fixed or random intervals in the same way that occasionol volume changes are included in a standard NPT ensemble simulation. For convenience, we pick a reaction at random after a fixed 
number of Monte Carlo steps. For a given state $r$, the number of ways to choc se a forward reaction is given by

$$
N_{f}(v)=\prod_{t=0}^{m}\left(\begin{array}{c}
M_{t} \\
\nu_{t}
\end{array}\right) .
$$

Similarly, the number of choices for the backward reaction is given by

$$
N_{b}(r)=\prod_{t=0}^{m}\left(\begin{array}{c}
M_{t} \\
\xi_{t}
\end{array}\right) .
$$

For a given chemical reaction step, we choose the forward path with probability $\frac{N_{1}}{N_{f}+N_{0}}$ and the backward reaction with probability $\frac{N_{f}}{N_{f}+N_{b}}$. Then the reactants are chosen randomly from the available molecules of the proper type. This is equivalent to choosing $\nu=\sum \nu_{i}$ particles at random and proceeding with the trial reaction if the set of molecules is that of either side of Equation (15). Otherwise, the process is repeated with another set of $\nu$ particles chosen at random until an acceptable set of particles is found.

Let $s$ denote a specific final state for the forward reaction and $t$, a specific final state for the reverse reaction. The final state molecule types are assigned randomly over the chosen $\nu$ particies for the reaction. The number of distinct final states for this process is $\nu ! / \prod \xi_{t}$ ! for the forward reaction and $\nu ! / \prod \nu_{t}$ ! for the reverse reaction where we have used $\nu=\xi$. So the un. weighted probability of choosing a move from state to state $s$ is given by

$$
p_{r \rightarrow 0}=\frac{\prod_{t=0}^{m} \xi_{t} !}{\nu !\left(N_{f}(r)+N_{b}(r)\right)} .
$$

Similarly,

$$
p_{s \rightarrow r}=\frac{\prod_{t=0}^{\dot{m}} \nu_{t} !}{\nu !\left(N_{f}(s)+N_{b}(s)\right)},
$$

and consequently

$$
\frac{p_{s \rightarrow r}}{p_{r \rightarrow 0}}=\frac{\prod_{t=0}^{m} \nu_{t} !\left(\left(N_{f}(r)+N_{b}(r)\right)\right.}{\prod_{t=0}^{m} \xi_{t} !\left(N_{f}(s)+N_{b}(s)\right)}
$$

and similarly for the reverse reaction. The acceptance probabilities for a chemical reaction move are then obtained from combining of Equation(11), Equation(14), Equation (20), and analogous results for the reverse reaction. These can be compared with a specific case given in reference 2 . For spatial moves not involving a chemical reaction, the usual canonical ensemble probabilities are used. In the isothermal-isobaric ensemble, mover in volume are also allowed in the standard fashion.

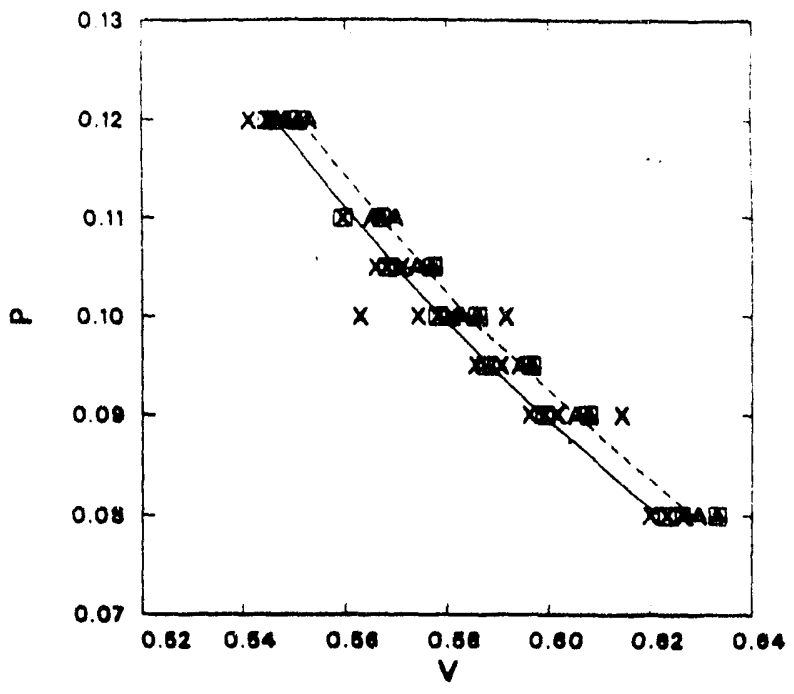

FIGURE 1. Pressure vs. Volume for Various Values of $\mathrm{T}$. Simulations (X) and interpolated Hugoniot (line) for the base case. Simulations (A) and interpolated Hugoniot (dashed line) for the A set of potentials.

\section{RESULTS}

Monte Carlo simulations have been performed in the atomic-isothermal-isobaric ensemble using the new method described above. A series of simulations have been made with nitrogen/axygen mixtures at high $P$ and T. Allowed molecular species are $\mathrm{N}_{2}, \mathrm{O}_{2}$, and $\mathrm{NO}$. The contribution to $W$ from $q_{i}$ 's and $\Lambda_{i}$ 's is given using constants and expressions found in McQuarrie ${ }^{5}$ and supplemented with electronic level constants 6,7 .

Initial conditions are a random set of positions for 120 molecules. Only $\mathrm{N}_{2}$ and $\mathrm{O}_{2}$ molecules are allowed in the initial configuration. The potentials are of the exponential-six form, $\phi=\epsilon\left(6 \exp \left(\alpha\left(1-r / r^{*}\right)\right)-\right.$ $\left.\alpha\left(r / r^{*}\right)^{-6}\right) /(\alpha-6)$, using the previously published ${ }^{2,3}$ parameters. Runs of $10^{6}$ steps were made with the first $2 \times 10^{5}$ steps ignored for the purpose of evaluating averages of quantities. Atomic exchanges were attempted every 37 steps and volume changes every 120 steps.

In this study we have expanded the set of calculated states to include the region near the $C J$ conditions for the detonation of liquid NO. This is the first time a CJ state has been calculated by an (essintially) exact method such as a Monte Carlo simulation. Statistical uncertainties from the Monte Carlo simulations are $\mathrm{E} \pm .00007 \mathrm{Mbar}-\mathrm{cm}^{3} / \mathrm{g}, \mathrm{V} \pm .00040 \mathrm{~cm}^{3} / \mathrm{g}, \mathrm{u}_{s} \pm 7 \mathrm{~m} / \mathrm{s}$, $u_{p} \pm 2 \mathrm{~m} / \mathrm{s}$, and $x_{N O} \pm .002$ where $x_{N O}$ is the mole frac- 


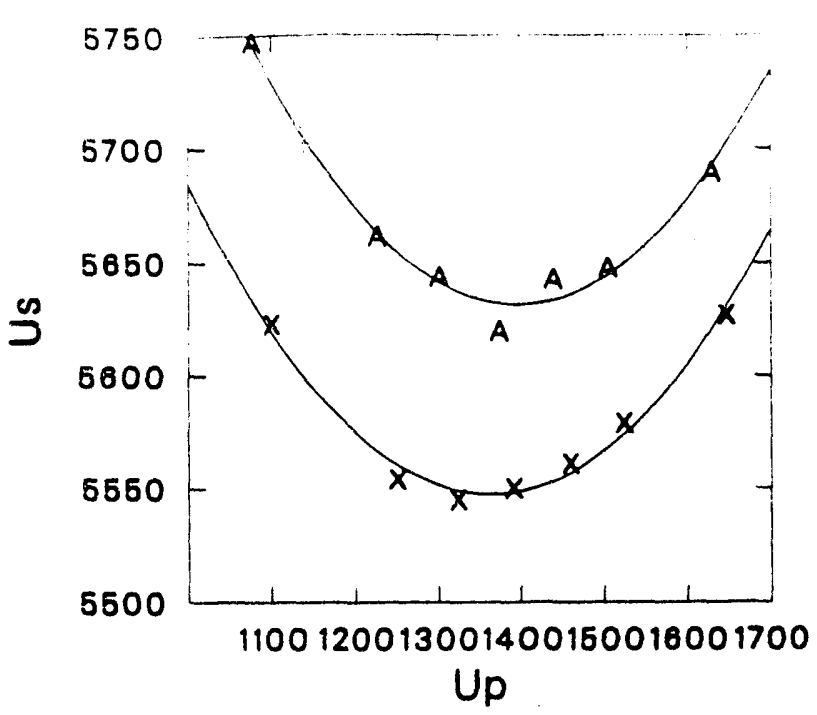

FIGURE 2. Shock Velocity vs. Particle Velocity.

tion of NO in the products. The Hugoniot conditions at a fixed pressure are found by interpolation (over $\mathbf{E}$ and $\mathrm{V}$ as a function of $\mathrm{T}$ ) between simulations to satisfy the jump condition, $E_{H}=E_{0}+\frac{1}{2} P\left(V_{0}-V\right)$. The initial conditions are taken as $\rho_{0}=1.294 \mathrm{~g} / \mathrm{cm}^{3}$ and $E_{0}=19.03$ $\mathrm{kcal} / \mathrm{mol}$ relative to $N_{2}$ and $\mathrm{O}_{2}$ at $\mathrm{T}=0$ and $\mathrm{P}=0$. The simulation data is plotted in Figure 1 in the form $P$ versus $V$. Simulations at $T=2500 \mathrm{~K}$ have a box drawn around the symbol. Except for a few simulations at $2000 \mathrm{~K}$ and $3000 \mathrm{~K}$, the data are at $100 \mathrm{~K}$ intervals. The symbol $X$ denotes the base case of potentials ${ }^{2,3}$ and $A$ denotes a set of potentials ${ }^{2,3}$ that differs slightly for one of the cross potentials. Note that the results for the base case and the $A$ case are significantly shifted relative to one another.

\section{DISCUSSION}

These results include the conversion of $P, V$ to $u_{s}, u_{p}$ from the standard formulae $u_{s}=V_{0} \sqrt{\frac{P-P_{p}}{V_{0}-V}}$ and $u_{p}=\sqrt{\left(P-P_{0}\right)\left(V_{0}-V\right)}$ and conversion to units of meters per second. The $u_{0}$ vs. $u_{p}$ data are shown in Figure 2 along with least square fits of a quadratic to the data. Note that the small change in cross potentials leads to almost $100 \mathrm{~m} / \mathrm{s}$ difference in detonation velocities. This change is due to a combination of changes in the EOS per se and changes in the equilibrium composition for the products.

We have demonstrated a new method for the calculation of the CJ state of an explosive using a set of Monte Carlo simulations giving directly the equilib-

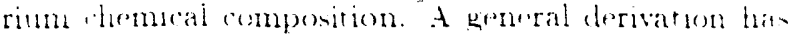
been given which allows for multiple species, multiple chemical reactions, and reactions involving a change in the total number of moles. The precision of the detonation velocity is a few meters per second for reasonable length simutations. Future application will be made of the method to more standard explosives. The extension of the method to the inclusion of solid products such as carbon is straightforward. Likewise, the method is easily extended to include fluid-fluid phase segregation (e.g. oil and water) utilizing the Gibbs ensemble method ${ }^{8}$ as has already been demonstrated ${ }^{9}$. The net result of this combination of methods will be to allow essentially exact calculation of detonation products EOS and CJ conditions for any given set of intermolecular potentials with a given free energy representation of solid products.

\section{ACKNOWLEDGEMENTS}

This work is supported by the U. S. Department of Energy.

\section{REFERENCES}

(1) M. P. Allen and D. J. Tildesely, Computer Simula tion of Liquids, Oxford: Clarendon Press, 1987.

[2] M. S. Shaw, J. Chem. Phys. 94, pp. 7550-7553 (1991).

[3] M. S. Shaw, "Direct Monte Carlo Simulation of Chemical Equilibrium Composition of Molecular Fluid Mixtures Under Shock Conditions" in Shock Compression of Condensed Matter - 1991, 1992, pp.131-134.

[4] N. Metropolis, A. W. Rosenbluth, M. N. Rosenbluth, A. H. Teller, and E. Teller, J. Chern. Phys. 21, pp. 1087-1092 (1953).

[5] Donald A. McQuarie, Statistical Mechanics, New York: Harper \& Row, 1976, ch. 6.

[6] M. W. Chase, Jr., C. A. Davies, J. R. Downey, Jr. D. J. Frurip, R. A, McDonald, and A. N. Syverud, "JANAF Thermochemical Tables, 3rd Edition", $J$. Phys. Chem. Ref. Data, 14, Suppl. 1 (1985).

[7] K. P. Huber and G. Herzberg, Molecular Spectra and Molecular Structure IV. Constants of Diatomic Molecules, New York: Van Nostrand Reinhold Co., 1979.

[8] A. Z. Panagiatopoulos, Mol. Phys. 61, pp. 813-826 (1987).

[9] J. K. Johnson, A. Z. Panagiatopoulos, and K. E. Gubbins, to be published. 

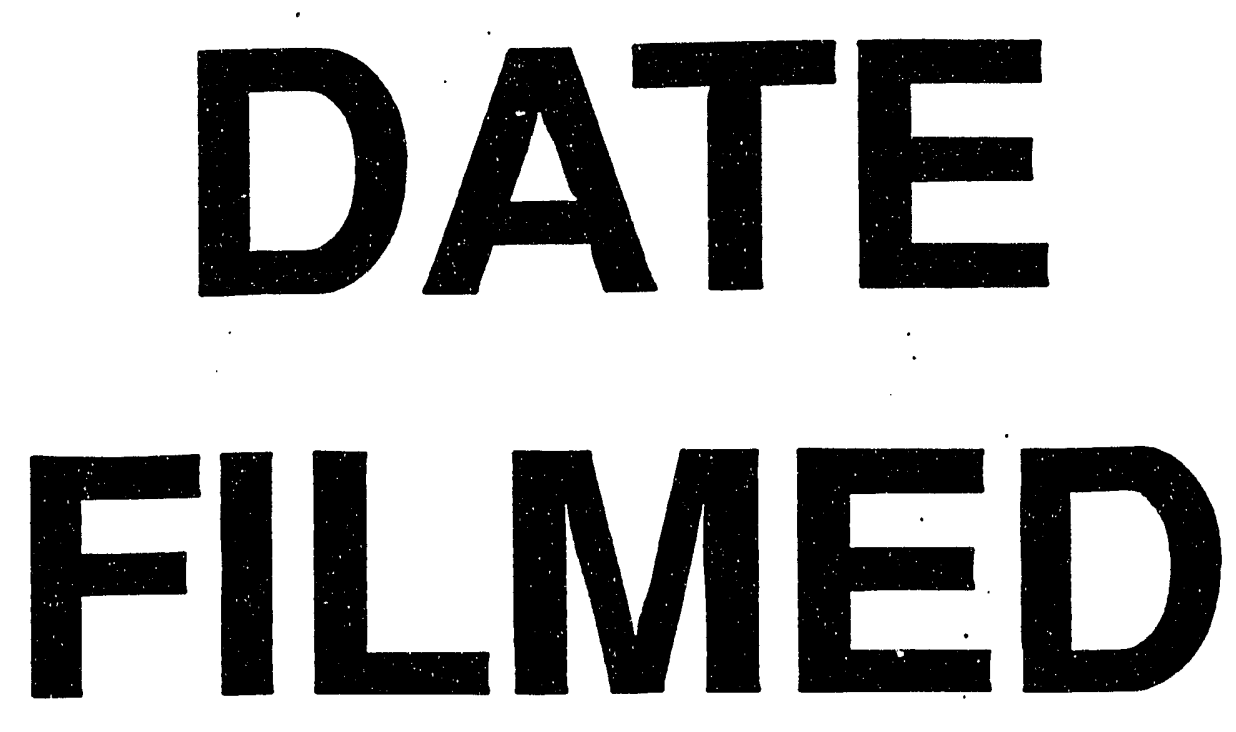

$11 / 4 / 93$
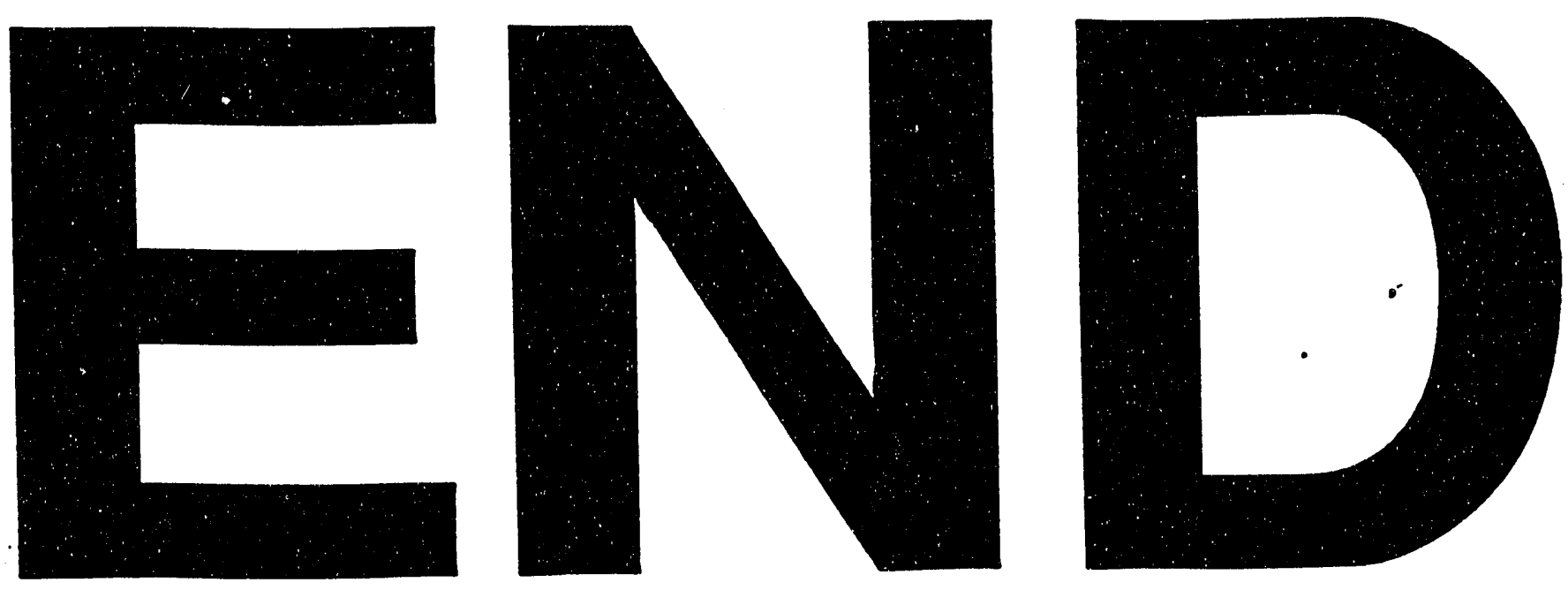
